\title{
Heinrich Schepers (1925-2020), In Memoriam
}

Griselda Gaiada

Heinrich Schepers falleció en la noche del 31 de diciembre de 2019 al primero de enero de 2020. Había cumplido noventa y cuatro años unos pocos días antes, la víspera de Navidad. Esa noche nos decía adiós uno de los mayores filósofos leibnizianos de todos los tiempos.

Schepers creció en el barrio de Belgrano, en Buenos Aires. Nuestro país, al que siempre evocaba con gran cariño, le dio el conocimiento del castellano que jamás olvidaría y seguiría hablando, pese al paso del tiempo, con perfecto acento rioplatense. En 1945, al terminar la guerra, volvió a su Alemania natal. Recaló en Münster, por entonces en ruinas. Con veinte años, ayudó a reconstruir la ciudad con sus propias manos, al tiempo que iniciaba sus estudios universitarios en Filosofía y Matemática. En 1957, se doctoró en la Universidad de Münster con una tesis sobre Andreas Rüdiger, ${ }^{1}$ bajo la dirección de Erich Hochstetter. Más tarde, en 1971, obtendría su habilitación con una investigación sobre dos dominicos, Robert Holkot y William Crathorn, pertenecientes a la primera generación de filósofos de Oxford después de Ockham. ${ }^{2}$ A partir de 1977 se desempeñó como profesor en la Universidad de Münster, donde en 1980 se le acordaría el cargo de profesor ordinario.

Gracias a la iniciativa de Erich Hochstetter, que en 1956 había fundado un instituto destinado a la edición de la correspondencia y de los escritos filosóficos de Leibniz (series II y VI de la Akademie-Ausgabe), Schepers se incorporó desde el inicio a este proyecto. El instituto devino la LeibnizForschungsstelle y, tras la muerte de Hochstetter en 1968, Heinrich Schepers tomó su dirección. El esmero que mostró en su labor de edición de la obra de

\footnotetext{
1 Andreas Rüdigers Methodologie und ibre Voraussetzungen. Ein Beitrag zur Geschichte der deutschen Schublphilosophie im 18. Jahrbundert. Köln, Kölner Universitäts-Verlag, 1959, 134 p. (Kantstudien, Ergänzungshefte 78).

${ }^{2}$ Holkot contra dicta Crathorn. I. Quellenkritik und biographische Auswertung der Bakkalaureatsschriften zweier Oxforder Dominikaner des XIV. Jahrhunderts », seguido de « Holkot contra dicta Crathorn. II. Das 'significatum per propositionem'. Aufbau und Kritik einer nominalistischen Theorie über den Gegenstand des Wissens ", Philosophisches Jahrbuch [der Görres-Gesellschaft], 77, 1970 et 79, 1972.
} 
Leibniz fue excepcional, como se aprecia en los volúmenes de la serie VI que estuvieron a su cuidado. Destaca, sin duda, el volumen 4 de dicha serie, que le insumió a él y a su equipo de Münster largos años de trabajo denodado. Precedido de diez Faszikel anuales (1982-1991) que constituyeron la Vorausedition ad usum collegialem, el volumen se publicó en 1999. El mismo, una joya editorial, incluye 3000 páginas de escritos filosóficos de Leibniz y varios cientos de páginas de aparato crítico.

Como buen leibniziano amante de la innovación, Heinrich Schepers fue además pionero en el uso de herramientas digitales, tomando la iniciativa de adoptar, ya en 1980, el software TUSTEP para facilitar la edición de los escritos de Leibniz en la Forschungsstelle de Münster. Quizá no resulte fácil a quienes no han tenido la experiencia del trabajo de edición dimensionar lo que su tarea ha significado. Esta implica, por supuesto, el desciframiento de los manuscritos, cosa que, tratándose de Leibniz, tenaz grafómano siempre a la búsqueda de la eficacia verbal, deviene ardua al extremo -caligrafía por momentos casi ilegible, escritos recurrentemente tachonados y sobrescritos, o fragmentados en trozos de papel que deben ser reconstituidos con ayuda de programas informáticos-. A este afanoso primer paso sucede luego la tarea de transcribirlos, de confeccionar la presentación tipográfica de todas las variantes, de establecer los textos definitivos, así como de datarlos según la cronología más verosímil, hecho que exige un profundo conocimiento del estilo, del vocabulario, del significado de los conceptos y, desde luego, de la evolución de todos ellos. Lo de Schepers fue, pues, un trabajo de hormiga, a la vez minucioso y titánico, del que todos los estudiosos de Leibniz somos encarecidamente deudores.

Es una obviedad tener que recordar que todo trabajo de edición es siempre hermeneusis, pero, tal como Schepers lo practicó, fue no sólo bermeneusis philosophica, sino también cabal comprensión de los principios, lo que constituye ni más ni menos que la esencia misma de la filosofía. Esto se tradujo en sus numerosos estudios dedicados a los aspectos cruciales de la filosofía de Leibniz. Los principales de ellos fueron compilados en su libro Leibniz. Wege zu seiner reifen Metaphysik, hito obligado para quienes estén iniciando su camino en Leibniz, pero además, y sobre todo, para quienes se precien de llamarse "leibnizianos". Las publicaciones posteriores a este libro ${ }^{3}$-de las que aquí

3 A saber: „Die sich selbst und ihre Welt konstituierenden Monade“ (deutschenglisch-französisch-spanisch). Anlässlich des 90. Geburtstages von H. Schepers hrsg. 
ofrecemos la traducción de "Iter rationis"- son igualmente expresión de la profundidad filosófica de Heinrich Schepers, a la que se añade el gran mérito de la llaneza expositiva. No está de más llamar la atención sobre la valía de este rasgo: el arte de la claridad, cuando va acompañado de honda penetración intelectual, es quizá uno de los mayores logros filosóficos, que sólo una lectura inadvertida se vería impedida de saber apreciar. Schepers supo conjugar "forma" y "fondo" al servicio de la vera philosophia.

Director infatigable de la Leibniz-Forschungsstelle hasta 1996, seguiría trabajando en ella hasta sus últimos días para fortuna de los investigadores que la frecuentaron. Todos aquellos que lo trataron coinciden en destacar la sabiduría, modestia, generosidad y hospitalidad que lo caracterizaron. A esto se podría agregar la "misteriosa intimidad" que el editor traba, inexorablemente, con la escritura y el pensamiento de aquél a quien ha consagrado la jornada entera de una vida. El providencial azar me permitió conocerlo hace ya varios años. Sus enseñanzas exceden el propósito de este exiguo homenaje, no así la más sentida de ellas, que su sonrisa luminosa volvía singularmente manifiesta y que él mismo, parafraseando a Bertolt Brecht, sintetizaría en vida, sentimiento y obra como sigue: die leben im Lichte, uns im Schatten sieht man nicht. ${ }^{4}$

von W. Li. Hefte der Leibniz-Stiftungsprofessur, Bd. 28. Hannover: Wehrhahn Verlag, 2016, 51 p.; „Leibniz’ unverstandene, aber gut zu verstehende Metaphysik“. Aus: X. Internationaler Leibniz-Kongress. "Für unser Glück oder das Glück anderer ». Hannover, 18.-23. Juli 2016. Band 6. Hildesheim, Georg Olms Verlag, 2017, pp. 227-242; „Iter rationis. Reise der Vernunft in Leibniz' Welt der Monaden“. Studia Leibnitiana. 49, 2017/1, p. 2-27; Leibniz frühe und dauernde Metaphysik. Sein Brief an Wedderkopf mit Konsequenzen. Studia Leibnitiana. 49, 2017/2, pp. 130-138.

${ }^{4}$ „Leibniz: Fiktion und Wahrheit“, Leibniæ: Wege zu seiner reifen Metaphysik, Berlin, Akademie Verlag, p. 269. 


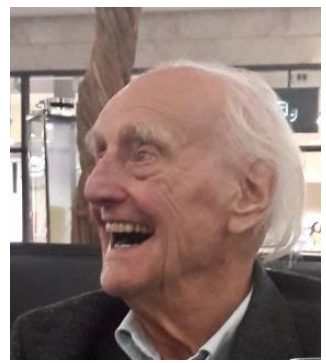

Münster, 17 de octubre de 2019

\section{Bibliografía de los estudios leibnizianos de Heinrich Schepers ${ }^{5}$}

\section{$\therefore \quad$ Escritos compilados por Heinrich Schepers en su libro Leibniz. Wege zu seiner reifen Metaphysik. Berlin, Akademie Verlag, 2014, 352 páginas:}

1. Möglichkeit und Kontingenz. Zur Geschichte der philosophischen Terminologie vor Leibniz. Filosofia (Torino) 14/4, Suppt. (1963), pp. 901-914.

2. Zum Problem der Kontingenz bei Leibniz. Die beste der möglichen Welten. In Collegium Philosophicum. Studien Joachim Ritter zum 60. Geburtstag. Basel-Stuttgart, Schwabe \& Co. Verlag, , 1965, pp. 326-350.

3. Glück durch Wissen. Zur Bestimmung des Philosophen durch Leibniz. Archiv für Begriffsgeschichte. XXVI (1982), Heft 2, pp. 184-192.

4. Leibniz' Arbeiten zu einer Reform der Kategorien. Zeitschrift für philosophische Forschung, 20 (1966), Heft 3 u. 4, pp. 539-564.

5. Demonstrationes Catholicae - Leibniz' großer Plan. Ein rationales Friedensprojekt für Europa. In: Pluralität der Perspektiven und Einheit der Wabrheit im Werk von G. W. Leibniz. Beiträge zu seinem philosophischen, theologischen und

\footnotetext{
${ }^{5}$ Este listado de bibliografía fue extraído del texto a la memoria de Schepers redactado por Michel Fichant para el Bulletin leibnizien VI de la Société d'études leibnizziennes de langue française, a publicarse en julio de 2020. Invito a leer también el bello homenaje que le brindó en ocasión de sus 90 años: Michel Fichant, «Célébration du 90 ème anniversaire du Professeur Dr. Heinrich Schepers, Hanovre, 18 février 2016 - Laudatio », Studia Leibnitiana, 48, 2016/1, p. 2-5.
} 
politischen Denken. Hrsg. v. F. Beiderbeck u. St. Waldhoff. Berlin, Akademie Verlag, 2011, pp. 3-14.

6. Die Polaritäten des Einen und des Vielen im Begriff der Monade. In Unita e molteplicità nel pensiero filosofico e scientifico di Leibniz. Simposio internazionale, Roma, 3-5, Oktober 1996. Hrsg. v. A. Lamarra u. R. Palaia, Firenze, Olschki (Lessico lntellettuale Europeo), 2000, pp. 171-184.

7. Gedanken zu den Philosophischen Schriften (Vl, 4). In Gottfried Wilhelm Leibniz, Sämtliche Schriften und Briefe. Hrsg. von der BerlinBrandenburgischen Akademie der Wissenschaften und der Akademie der Wissenschaften in Göttingen. Reihe VI: Philosophische Schriften, hrsg. von der Leibniz-Forschungsstelle der Universität Münster. Bd. 4, Berlin, Akademie Verlag, 1999, pp. XLV-XCI.

8. Schwierigkeiten mit dem Körper. Leibniz' Weg zu den Phänomenen. In VII. Internationaler Leibniz-Kongress. Nibil sine ratione. Natur und Technik im Wirken von G. W. Leibniz. TU Berlin, 9.-15. September 2001. Nachtragsband. Hannover, 2002, pp. 99-110.

9. "De affectibus". Leibniz an der Schwelle zur Monadologie. Studia Leibnitiana. 35, 2003, p. 133-161.

10. Wurzeln und Austriebe des metaphysischen Rationalismus bei Leibniz. In Les enjeux du rationalisme moderne: Descartes, Locke et Leibniz. Coloquio organizado por Tahar Ben Guiza en Beit al-Hikma (Khartago, 24.-27. Januar 2004), Tunis, 2007, pp. 291-299.

11. Ein Plädoyer für Leibniz' strenge Rationalität. Einspruch gegen die behauptete Gleichrangigkeit von sanfter und strenger Rationalität. In Logic, Epistemology, and the Unity of Science. Bd. 13. Hrsg. v. M. Dascal, Springer Science + Business Media 2009, pp. 17-35.

12. Leibniz' Konzeptualismus. Ein Programm. [Workshop: Leibniz and the Scholastic Tradition. Pisa, Scuola Normale Superiore, 6.-8. Juni 2005].

13. Perzeption und Harmonie. In VIII. Internationaler Leibniz-Kongress. Einheit in der Vielheit. Hannover, 24.- 29. Juli 2006. Nachtragsband, hrsg. v. H. Breger, Hannover 2007, pp. 200-216.

14. Leibniz: Fiktion und Wahrheit. In Science Fiction im Barock. Utopisches Denken von Roger Bacon bis Gottfried Wilhelm Leibniz. Beiträge zur Tagung auf Schloss Hundisburg vom 15. bis 17. Mai 2009. Hrsg. von Berthold Heinecke. Berlin, Weidler, 2013, pp. 225-239. 
15. Neues über Zeit und Raum. Studia Leibnitiana. Sonderheft 37, 2010, pp. 3-18.

16. Ist unsere die beste der möglichen Welten? Was fordert Leibniz zur Affirmation seiner These? Rechtstheorie, 42, 2011-1, pp. 1-20.

17. Der rationale Kern der Theodizee. Internationales Symposium der Leibniz-Edition Potsdam der Berlin-Brandenburgischen Akademie der Wissenschaften. In 300 Jahre Essais de Théodicée. Rezeption und Transformation. Hrsg. v. W. Li. und W. Schmidt-Biggernann. Studia Leibnitiana. Supplementa Bd. 36, 2013, pp. 23-35.

18. Die Natur des Subjekts und das Subjekt der Natur. In IX. Internationaler Leibniz-Kongress. Natur und Subjekt. Hannover, 26. September bis 1. Oktober 2011. Vorträge. 3. Teil, hrsg. v. H. Breger, Hannover 2011, pp. 1009 1023.

\section{$\therefore \quad$ Otros estudios:}

19. Begriffsanalyse und Kategorialsynthese. Zur Verflechtung von Logik und Metaphysik bei Leibniz. In Akten des [I.] Internationalen Leibniz-Kongresses. Hannover 14.-19. November 1966. Bd. III, Erkenntnistheorie-LogikSprachphilosophie-Editionsberichte. Studia Leibnitiana. Supplementa Bd. 3.3, pp. 34-49.

20. Leibniz' Disputationen 'De Conditionibus'. Ansätze zu einer juristischen Aussagenlogik. In Akten des II Internationalen Leibniz-Kongresses. Hannover 17.-19. November 1972. Bd. IV, Logik-ErkenntnistheorieMethodologie-Sprachphilosophie. Studia Leibnitiana. Supplementa Bd.15, p. 117.

21. Spiritus bei Leibniz. In Spiritus. IV Colloquio Internazionale. Roma 79 gennaio 1983. Atti a cura di Marta Fattori, Massimo Bianchi. Roma, Edizioni dell'Ateneo, Roma 1984 (Lessico intelletuale europeo 32).

22. Scientia Generalis. Ein Problem der Leibniz-Edition. In $V$. Internationaler Leibniz-Kongress. Leibniæ. Tradition und Aktualität. Hannover, 14.-18. November 1988. Vorträge II. Teil, Hannover 1989, pp. 350-359.

23. Die sich selbst und ihre Welt konstituierenden Monade (deutschenglisch-französisch-spanisch). Anlässlich des 90. Geburtstages von H. Schepers, hrsg. von W. Li. Hefte der Leibniz-Stiftungsprofessur, Bd. 28. Hannover, Wehrhahn Verlag, 2016, 51 p. 
24. Leibniz' unverstandene, aber gut zu verstehende Metaphysik. Aus $X$. Internationaler Leibniz-Kongress. "Für unser Glück oder das Glück anderer». Hannover, 18.-23. Juli 2016. Band 6. Hildesheim, Georg Olms Verlag, 2017, pp. 227-242.

25. Iter rationis. Reise der Vernunft in Leibniz' Welt der Monaden. Studia Leibnitiana. 49, 2017/1, p. 2-27.

26. Leibniz frühe und dauernde Metaphysik. Sein Brief an Wedderkopf mit Konsequenzen. Studia Leibnitiana. 49, 2017/2, p. 130-138.

$\therefore \quad$ Entradas al Historisches Wörterbuch der Philosophie. Hrsg. Von Joachim Ritter, Basel-Stuttgart, Schwabe 1971-2004:

Allgemeines / Besonderes - A priori / a posteriori - Complementum possibilitatis - Enthymem - Epicherem - Episyllogismus / Prosyllogismus Eselbrücke - Existentificatio - Heuristik - Inventio medii Kohaesion / Kohärenz - Konkomitanz - Konvenienz - Logica vetus / Logica nova, Logica antiqua / Logica modernorum - Obligatio, Ars obligatoria - Philosophie: Leibniz - Quadrat, logisches - Scientia generalis Transkreation - Vernunftwahrheiten / Tatsachenwahrheiten - Verträglichkeit, Kompatibilität - Zentralmonade. 